\title{
M. M. POSTNIKOV: HIS LIFE, WORK AND LEGACY
}

\author{
YULI RUDYAK \\ Department of Mathematics, University of Florida \\ 358 Little Hall, Gainesville, FL 32611, U.S.A. \\ E-mail:rudyak@math.ufl.edu
}

Mikhail Mikhailovich Postnikov was born on October 27, 1927. In 1987, on the occasion of Postnikov's 60th birthday, several of his students decided to write an article about him. One of them, Andrey Khokhlov, recorded Postnikov's narration of his life. The recording occupies three audio cassettes; the participants of the conference had a chance to listen to a small part of it. These notes, as well as the papers [2, 1, 25], are partially based on that recording.

If you look at the date of Postnikov's birth, 27.10 .1927 (or 10/27/1927 in the United States), you will see that it is almost a "lucky number", that is, the sum of the first four digits equals the sum of the last four (almost, since we accept the usual agreement $9=0$ ). According to a popular belief, this does not necessarily mean that Postnikov was born with a silver spoon in his mouth, but rather that he would overcome most troubles in his life. Once I told this to Postnikov, and he replied, "Oh well, this is exactly the way my life is." You will see examples of this below, see also [25, especially pages 167, 168].

Postnikov was born in Shatura, a suburb of Moscow, where his father worked as an electrical engineer and his mother worked as a school teacher. A few years later the family moved to Perm. When Postnikov was 10 years old, his father was arrested and disappeared in the Gulag (he was exonerated later).

Postnikov learned how to read quite early, and during his childhood he was what Russians would call a "book worm" (книжный червь), a person who spends most of his time reading books. Because of this, as well as because of his talent, Postnikov was way ahead of most of his counterparts, and so the program of elementary school was too easy for him. However, he was not allowed to drop the school and was obligated to attend the boring classes. At that time he divided all his teachers into two categories: stupid and smart. The stupid ones always bothered him, by demanding that he listen to the teacher and not read books during the class. The smart teachers allowed him to read all

2000 Mathematics Subject Classification: Primary 55M99, 55S30; Secondary 68T40. Key words and phrases: Postnikov. 
the books he wanted, but there was an agreement: At any time the teacher could ask Postnikov to explain what was being discussed in the class, and in the case of failure he would get a bad grade (which never happened, I believe).

In 1942, Postnikov entered Perm University. According to Russian regulations, a person that wants to enter a university has to take entrance exams, and then the applicants with the best grades are admitted. Furthermore, only a person who completed ten years of school is allowed to take the entrance exams. However, Postnikov turned out to be an exception. Being just a bit less than 15 years old in September 1942, and having completed only eight years of school at the time, he got a special permission to take the entrance exams. The reason was, mainly, that this happened in a wartime, and most young men were not students but soldiers. Of course, his knowledge was good enough to pass the exams successfully, and in 1942 Perm University registered him as a new student.

At the university, Postnikov first enjoyed arranging his scrappy mathematical knowledge into a system, but in a short while he realized that most professors just followed the textbooks and explained nothing new. A nice exception was Sophia Yanovskaya, the Moscow State University professor that ended up in Perm after evacuation (remember that all this happened during wartime). Along with her, there was a third-year student Eugene Dynkin (in 2004 the Cornell University celebrated his 80-th anniversary). Interaction with these two people convinced Postnikov that a proper mathematical education could only be obtained at Moscow State University.

In 1943, Yanovskaya and Dynkin returned to Moscow. Postnikov wanted to follow them there, but there were many administrative obstacles to doing this. For example, because of the wartime, people were not allowed to visit Moscow without special permission, private citizens could not buy a train ticket to Moscow, etc. In addition, Postnikov was less than 16 at the time and so he could not obtain any ID.

Postnikov told that he had overcome all of these difficulties successfully, arriving in Moscow in a cold dilapidated railroad car. (There exist several legends about this trip but Postnikov never confirmed or repudiated them.) One way or another, in early November 1943, the sixteen year old Postnikov arrived (illegally!) in Moscow, at the Kursk railway station.

Postnikov knew only one Moscow inhabitant. It was Yanovskaya, and fortunately for him, he knew her address. It was a non-trivial task to find the house for a person who had never been to Moscow before, and naturally it took some time and effort. Eventually, at 11 o'clock at night, he knocked at the door of Yanovskaya's apartment. She opened the door. Being quite a bit surprised, she nevertheless welcomed him. She offered him some hot tea and a sofa to sleep (Postnikov always emphasized these two things, the hot tea and the sofa - after the railroad trip he had to endure.)

The next day, the two of them showed up at the Department of Mechanics and Mathematics of Moscow State University. Ivan Petrovskiu, who was the chair of the department at the time, welcomed Postnikov by saying "Professor Yanovskaya recommended you as a capable young man that wants to be our student. Well, please, take all the exams for the first year courses, take some of the exams for the second years courses. Then we'll see what kind of bird you are, and we'll make a decision." 
In the month of November, Postnikov did not have any papers, income, or dormitory room. Nevertheless, he quickly passed the exams, and in the beginning of December, was enrolled as a second year student on the order of Petrovskiu. In particular, he became a legal resident of Moscow.

Through those trying times, Postnikov was guided by his love for mathematics. He kept that romantic relationship with mathematics his entire life, being interested in all (not only topological) new results and ideas, always ready to engage in a discussion.

During his university years, Postnikov studied mathematics very intensely. In 1943-44 he passed not only the second year, but also the third year courses. In addition to the mandatory courses, he also passed 17 elective courses (the required number was only three). In the Summer of 1945, Postnikov graduated from the university with Master's degree with honors. At that time, he was only 18 years old.

The choice of a supervisor is a very important decision for any student who wants to become a scientist. Let me quote Postnikov [25] about this step of his biography.

"The assistant dean ... called me in to say ,'Postnikov, you need to have an advisor for your senior thesis. Whom do you want, Lusternik or Aleksandrov?'... And at this point a very strange thing happened. Without a moment's hesitation I blurted out, 'I want Lev Semenovich Pontryagin!'. The reason why this was so strange is that I did not know Pontryagin, and had only taken his very boring, formalistic course of homological algebra. Yet my spontaneous choice turned out to be an unusually good one: if it weren't Pontryagin it's hard to say what would become of me ... The day after my conversation with the assistant dean, seeing Pontryagin in the hall, I said to him 'Lev Semenovich! My name is Postnikov, I am a fifth-year student, and I told the dean that you are my advisor!' Pontryagin was startled, but he answered, 'Fine. Come to see me at home tomorrow, and we'll talk'. That is how I became Pontryagin's student."

For Postnikov's senior thesis, Pontryagin suggested that he investigate the intersection $\mathbb{Z} / 2$-rings of closed 3 -manifolds, to find a condition that would characterize the intersection rings of 3-manifolds that bound 4-manifolds. Postnikov found such a condition, but then he realized that every 3-manifold satisfies it! (Now we know that nothing else could happen, since every closed 3-manifold is a boundary [26].) In fact, Postnikov proved that, starting from the 3 -sphere and using Dehn surgery, one can obtain all possible intersection rings of 3-manifolds. This was the subject of the senior thesis that Postnikov defended in 1945 .

In the Fall of 1945, Postnikov became Pontryagin's PhD student. At that time, the central problem of algebraic topology was homotopy classification of spaces and maps. In other words, one wanted to tell whether two maps are homotopic (or two spaces are homotopy equivalent) in terms of some reasonable algebraic invariants. So, it is not surprising that Pontryagin was also interested in this circle of problems.

The first result in this area was probably the theorem of Brouwer [3] that states that the sphere is not contractible. Following this was the result that, for a closed orientable manifold $M^{n}$, the homotopy class of a map $M^{n} \rightarrow S^{n}$ is completely determined by its degree (Brouwer for $n=2$, Hopf for all $n$, see [5]). In particular, there are countably many maps $S^{n} \rightarrow S^{n}$. One could also mention Hopf's discovery [5] of a homotopically 
non-trivial map $S^{3} \rightarrow S^{2}$ (there is a countable infinity of such classes, classified by the so-called Hopf invariant), and Whitehead's homotopy classification of simply connected 4-dimensional polyhedra [28].

In 1933, Hopf [7] classified maps of an $n$-dimensional (finite) polyhedron $X^{n}$ to $S^{n}$. Namely, two maps $f, g: X^{n} \rightarrow S^{n}$ are homotopic if and only if the induced homomorphisms $f_{*}, g_{*}: H_{n}(X ; \mathbb{Z} / m) \rightarrow H_{n}\left(S^{n} ; \mathbb{Z} / m\right)$ coincide for all $m$.

With the development of the language of cohomology, Whitney [30] reproved the above mentioned classification theorem in the following form: Two maps $f, g: X^{n} \rightarrow S^{n}$ are homotopic if and only if the induced homomorphisms $f^{*}, g^{*}: H^{n}\left(S^{n}\right) \rightarrow H^{n}(X)$ coincide. Note that here we only use $\mathbb{Z}$-coefficients, unlike in Hopf's homology classification. This made it absolutely clear that cohomology is a more suitable apparatus for the classification problem than homology.

Whitney noticed that his method of homotopy classification of maps $X^{n} \rightarrow S^{n}$ can be used as well for homotopy classification of maps $X^{n} \rightarrow Y, n>1$, where $Y$ is an $(n-1)$-connected space, i.e. $\pi_{i}(Y)=0$ for $i<n$. Such a space $Y$ is (weakly) homotopy equivalent to a $\mathrm{CW}$ space with one 0 -cell and no $k$-cells with $0<k<n$. Then each $n$-cell $e$ gives us an element $a_{e} \in \pi_{n}(Y)$, and the correspondence $e \mapsto a_{e}$ yields a cohomology class $u \in H^{n}\left(Y ; \pi_{n}(Y)\right)$, called the fundamental class of $Y$. Now, the theorem states that two maps $f, g: X \rightarrow Y$ are homotopic if and only if $f^{*} u=g^{*} u$. Moreover, every class $a \in H^{n}\left(X ; \pi_{n}(Y)\right)$ can be realized as $f^{*}(u)$ with some $f$. In other words, there is a natural bijection between homotopy classes of maps $X \rightarrow Y$ and elements of $H^{n}\left(X ; \pi_{n}(Y)\right)$.

From now on, given two spaces $X$ and $Y$, we will use the notation $[X, Y]$ for the set of homotopy classes of maps $X \rightarrow Y$, and the symbol $\cong$ for isomorphism of groups, homotopy of maps, and homotopy equivalence of spaces.

The next step was the classification of maps of $(n+1)$-dimensional polyhedra $X$ to $S^{n}$. This was done by Pontryagin for $n=2$ and by Steenrod for $n>2$. Steenrod's result [27] can be stated in the form of the exact sequence

$$
H^{n-1}(X) \stackrel{\varphi}{\rightarrow} H^{n+1}(X ; \mathbb{Z} / 2) \rightarrow\left[X, S^{n}\right] \rightarrow H^{n}(X) \stackrel{\varphi}{\rightarrow} H^{n+2}(X ; \mathbb{Z} / 2)
$$

where $\varphi(x)=S q^{2}(x \bmod 2)$. It is worth noting that Steenrod introduced his famous operations in order to attack the above mentioned homotopy classification problem.

The result of Pontryagin [17] is more delicate since here we are in the unstable situation: $\left[X, S^{n}\right]$ is not a group for $\operatorname{dim} X=3$ and $n=2$. It is still possible to write an "exact sequence" as above, using a certain unstable cohomology operation (Pontryagin square), but the map $f:\left[X, S^{2}\right] \rightarrow H^{2}(X)$ is not a homomorphism, and we cannot say that the inverse images of elements of $H^{2}(X)$ have the same cardinality. For example, if $X=S^{1} \times S^{2}$ and $d \in \mathbb{Z}=H^{2}(X), d \neq 0$, then $f^{-1}(d)$ has $2|d|$ elements.

In his $\mathrm{PhD}$ thesis, Postnikov classified maps of 3-dimensional polyhedron to a simply connected space, [18]. Later he extended this result and classified maps of an $(n+1)$ dimensional polyhedron $X$ to an $(n-1)$-connected space $Y$, see [19]. As you can see, his results are a mixture of the Pontryagin-Steenrod and Whitney type results.

In general, one can say that the complexity of the classification problem is determined by the difference between the dimension of the domain and the connectedness of the range, 
that is, if you investigate maps $X \rightarrow Y$ where $\operatorname{dim} X=k$ and $Y$ is $(n-1)$-connected, then the number $k-n+1$ can be regarded as the complexity of the problem. From this point of view, the Hopf-Whitney theorem solves a problem of the first level of complexity, while the above mentioned problems solved by Pontryagin, Steenrod and Postnikov are of the second level of complexity.

Right after defending his PhD thesis in 1949, Postnikov started his job in Pontryagin's department at the Steklov Mathematical Institute. He used the ideal research conditions by working intensively and, during the next two years, he finished what was to become the main accomplishment of his mathematical career: the homotopy classification of polyhedra and their maps. Let me discuss this in greater detail.

With the development of the obstruction theory, [8], it was realized that the Whitney Classification Theorem can be restated as follows: Let $Y$ be a space such that $\pi_{i}(Y)=0$ for $i \neq n, n>1$, and let $X$ be an arbitrary polyhedron. Then the map $[f] \mapsto f^{*}(u)$ yields a natural bijection $[X, Y] \cong H^{n}\left(X ; \pi_{n}(Y)\right)$. Yet another way to state the above theorem is to define for every $n>1$ and every abelian group $\pi$ the Eilenberg-MacLane space $K(\pi, n)$ as a CW complex such that $\pi_{i}(K(\pi, n))=\pi$ for $i=n$ and is trivial otherwise. Then $[X, K(\pi, n)] \cong H^{n}(X ; \pi)$.

From this point of view, the complexity of the classification problem for maps $X \rightarrow Y$ is measured by the number of non-trivial homotopy groups of $Y$ : The simplest case is when $Y$ is an Eilenberg-MacLane space, the problems of the second level complexity appear when $Y$ has exactly two non-trivial homotopy groups, etc. This is because, when you classify maps $X \rightarrow Y$ with $\operatorname{dim} X=k$, the groups $\pi_{i}(Y), i>k$, are out of play. It is not surprising that the homotopy complexity of a space can also be measured by the number of its non-trivial homotopy groups. Indeed, by the Universal Coefficients Theorem, $[K(\tau, n), K(\pi, n)] \cong \operatorname{Hom}(\tau, \pi)$, and this implies easily that the homotopy type of a CW complex $K(\pi, n)$ is completely determined by $\pi$ and $n$.

The homotopy groups, however, do not determine the homotopy type of a space. For example, the spaces $S^{2}$ and $S^{3} \times \mathbb{C P}^{\infty}$ have the same homotopy groups but different homology (and hence different homotopy type). Hence, one can pose the following problem. Given two polyhedra with isomorphic homotopy groups, what else do we need in order to ensure that these polyhedra are homotopy equivalent? In other words, given a polyhedron with given homotopy groups, what else do we need in order to determine its homotopy type completely?

Postnikov solved this problem. As we have already noticed, in case of only one nontrivial homotopy group, the homotopy type of a CW complex is completely determined by this group. Generally, however, the information on homotopy groups is not enough: we need countably many invariants discovered by Postnikov and now known as the Postnikov invariants. Homotopy groups together with Postnikov invariants completely determine the homotopy type of a finite dimensional CW complex. (For infinite dimensional CW complexes, one can only claim that the spaces have the same $n$-type for all $n$, but this does not imply that the spaces are homotopy equivalent, see [29].)

The contemporary approach to the Postnikov theory is as follows. First, consider a (simply connected) CW space $Y$ with only two non-trivial homotopy groups $\pi$ and $G$ in 
dimensions, $m$ and $n$, respectively, $m<n$. Then, following Serre, we construct a fibration $p: Y^{\prime} \rightarrow K(\pi, m)$ where $Y^{\prime}$ is homotopy equivalent to $Y, K(\pi, m)$ is an EilenbergMacLane space, and $p_{*}: \pi_{m}\left(Y^{\prime}\right) \rightarrow \pi_{m}(K(\pi, m))$ is an isomorphism. The fiber of this fibration is (homotopy equivalent to) $K(G, n)$. Let $u \in H^{n}(K(G, n) ; G)$ be the fundamental class. Consider the transgression $\tau: H^{n}(K(G, n) ; G) \rightarrow H^{n+1}(K(\pi, m) ; G)$ and put $\kappa=\tau u \in H^{n+1}(K(\pi, m) ; G)$, that is, let $\kappa$ be the characteristic class of the fibration. Then the quintuple $(m, n ; \pi, G ; \kappa)$ completely determines the homotopy type of $Y$, i.e. two CW spaces with the same quintuples are homotopy equivalent.

Concerning the classification problem, note that the isomorphism $[X, K(G, n+1)] \cong$ $H^{n+1}(X ; G)$ allows us to regard the class $\kappa$ as a (homotopy class of the) map $K(\pi, m) \rightarrow$ $K(G, n+1)$, i.e. as a cohomology operation $\kappa: H^{m}(-; \pi) \rightarrow H^{n+1}(-; G)$. Then we have an exact sequence

$$
H^{m-1}(X ; \pi) \stackrel{\Omega \kappa}{\longrightarrow} H^{n}(X ; G) \longrightarrow[X, Y] \longrightarrow H^{m}(X ; \pi) \stackrel{\kappa}{\longrightarrow} H^{n}(X ; G) .
$$

In the stable case, when $\operatorname{dim} X<2 m-1$, this is an exact sequence of groups, and the group $[X, Y]$ can be computed from it up to extension.

More generally, we can proceed by induction. Given a $\mathrm{CW}$ space $Y$ and a number $n$, there exists a map $p_{n}: Y \rightarrow Y_{n}$ such that $\pi_{i}\left(Y_{n}\right)=0$ for $i>n$ and $p_{*}: \pi_{k}(Y) \rightarrow \pi_{k}\left(Y_{n}\right)$ is an isomorphism for $i \leq n$. Changing $Y_{n}$ within its homotopy type if necessary, we get a commutative diagram (Postnikov tower)

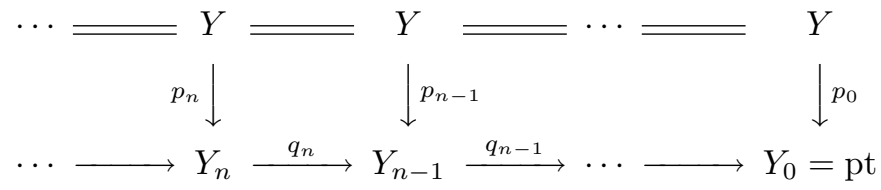

where each map $q_{n}$ is a fibration whose fibers have the homotopy type of the EilenbergMacLane space $K\left(\pi_{n-1}(Y), n\right)$. We denote by $\kappa_{n} \in H^{n+1}\left(Y_{n} ; \pi_{n-1}(Y)\right)$ the characteristic class of the fibration $q_{n}$. This class $\kappa_{n}$ is called the $n$-th Postnikov invariant of $Y$. The homotopy type of $Y_{n}$ turns out to be completely determined by the homotopy type of $Y_{n-1}$ and the class $k_{n}$. The homotopy groups and the Postnikov invariants together then determine the homotopy type of $Y$, because if $X$ and $Y$ are finite dimensional CW complexes and $X_{n} \cong Y_{n}$ for $n>\max \{\operatorname{dim} X, \operatorname{dim} Y\}$, then $X \cong Y$.

The above construction is natural, meaning that a map $X \rightarrow Y$ induces a map of commutative diagrams like the one above.

Finally, the classification problem can be attacked similarly to that with only two non-trivial homotopy groups, using the obvious induction along the Postnikov tower. Although the realization of this idea is quite complicated, and even the problems at the third level of complexity have not been completely sorted out, we still can say that the classification problem is solved "in principle".

Historically, the situation developed as follows. In the late 1940's, there was a great interest in relating the homology and homotopy groups. For example, as we already mentioned, if a space $X$ has only one non-trivial homotopy group $\pi$ in dimension $m$ then the homology of $X$ is completely determined by $\pi$ and $m$. In other words, if $X$ is a space 
whose homotopy groups $\pi_{i}(X)$ vanish for $i<m$ and $m<i<n$, then the homology groups $H_{i}(X), i<m$ are completely determined by $\pi_{m}(X)$ in a purely algebraic fashion. However, we cannot determine the group $H_{m}(X)$, even if we know the group $\pi_{m}(X)$, cf. the aforementioned example of the spaces $S^{2}$ and $S^{3} \times \mathbb{C P}^{\infty}$. To solve the problem, Eilenberg and MacLane $[9,10]$ introduced a new invariant $\kappa \in H^{n+1}\left(K\left(\pi_{m}(X), m\right) ; \pi_{n}(X)\right)$ and proved that the triple $\left(\pi_{m}(X), \pi_{n}(X), \kappa\right)$ determines the homology structure of $X$ in dimension $\leq n$ completely. They used the term " $k$-invariant" for $\kappa$.

Postnikov mastered the Eilenberg-MacLane ideas immediately and realized that the construction can be iterated, by constructing similar $k$-invariants for any pair of adjacent non-trivial homotopy group. This family of $k$-invariants is now known as the Postnikov invariants; the Eilenberg-MacLane $k$-invariant turns out to be the first non-trivial Postnikov invariant. Postnikov quickly wrote the paper [20] which emphasized the fact that the $k$-invariants completely determine the homology of a space. Later, however, he realized almost by accident that the $k$-invariants determine the entire homotopy type of a finite dimensional complex (see below notes about Postnikov's talk at the Kurosh seminar).

It is worth mentioning that Eilenberg and MacLane, as well as Postnikov, worked with simplicial sets, and that no such words as fibration, transgression, etc. appeared in their papers. The contemporary interpretation of $k$-invariants as characteristic classes of fibrations and the construction of Postnikov's tower of fibrations, as described above, are probably due H. Cartan and J.-P. Serre. Of course, Postnikov himself did not use the term "Postnikov system", he spoke about "natural system" or "homotopy resolution".

Postnikov published all of these results in the short articles [20, 21, 22]. Peter Hilton reviewed these papers and distributed them among Western topologists, with comments that he found these results very important. Because of this, Postnikov became famous at the West side of the "iron curtain", but still not in the USSR. Fortunately, in 1953 a delegation of British scientists, headed by John Bernal, visited Moscow. The outstanding British topologist J. H. C. Whitehead was among the delegates and, of course, Moscow mathematicians asked him to give a talk. He opened his talk in the Steklov Institute with the words "I apologize for bringing coal to Newcastle, but I will talk about the work of Postnikov, because it constitutes the greatest achievement in algebraic topology in recent years." In fact, he reformulated Postnikov results in terms of CW complexes. After this event, Postnikov was recognized as a top level topologist in the USSR as well.

The detailed version of Postnikov results was published in [23, 24]. In 1961, Postnikov was awarded the Lenin Prize, which was the highest scientific award in the USSR.

The following two stories offer a good example of communication between young and experienced mathematicians. Postnikov treated his system as follows: Suppose we have a finite CW complex $X$ with non-zero homotopy groups $\pi_{i}=\pi_{i}(X)$ in dimensions, say, $i=3,7,12$ only. In order to describe the homotopy type of $X$, we need the invariants $\kappa_{1} \in H^{8}\left(K\left(\pi_{3}, 3\right) ; \pi_{7}\right)$ and $\kappa_{2} \in H^{13}\left(X_{1} ; \pi_{12}\right)$. When Postnikov discussed this with Pontryagin, the supervisor suggested to change the numbering of $k$-invariants as follows: do not pay attention to whether a homotopy group is equal to zero or not, and let $\kappa_{i}$ be an element of the group $H^{i+1}\left(X_{i-1} ; \pi_{i}(X)\right)$. Now this numbering is commonly accepted. Last time when Postnikov recalled this (and it was more than 50 years after the fact), 
I still saw the fire in his eyes - so excited he was. "This remark of Pontryagin removed the fog from my eyes" ("Пелена слетела с моих глаз").

The other example: Postnikov gave a talk at the algebra seminar at Moscow State University. The seminar was run by A. G. Kurosh, the chairman of the Algebra Department. (He is well-known for his monograph on group theory.) The talk was entitled "How to compute homology groups of a space if we know its homotopy groups". Kurosh was not a topologist but he was a well-educated and broad mathematician, and he asked the question, "What other invariants of spaces can be computed using your $k$-invariants?" Postnikov thought a bit and answered, "You can compute any homotopy invariant. Moreover, you can determine the entire homotopy type." Of course, this changed the strength of Postnikov's results considerably.

The ideas of Postnikov were immediately exploited and developed, and they remain an active research topic today. For example, John Moore [16] proposed Postnikov systems over a space, meaning that a map $f: X \rightarrow B$ can be decomposed (up to homotopy) into a tower of fibrations

$$
\cdots \rightarrow X_{n} \stackrel{q_{n}}{\rightarrow} X_{n-1} \rightarrow \cdots \rightarrow X_{0}=B
$$

where $X_{n}$ has the $(n-1)$-type of $X$ and the fiber of each $q_{n}$ is an Eilenberg-MacLane space. This way, the homotopy fiber of $f$ is decomposed into a Postnikov system. The original Postnikov system corresponds to $B$ being a point. A stable version of the theory has also been developed, resulting in Postnikov systems for spectra.

It is worth mentioning that the Postnikov systems are a useful tool for constructing localizations of spaces, see [4]: It is clear how to localize an Eilenberg-MacLane space, and then one can perform the localization step by step, along the stages of the tower. In particular, the Sullivan model for the rational homotopy type of spaces [15] can be regarded as an algebraization of the Postnikov construction. Note that D. Sullivan's talk at this conference was entitled "Applications of algebraic analogues of Postnikov systems to geometry".

Outside of the homotopy theory, Postnikov collaborated successfully with M. A. Evgrafov on complex analysis and differential equations, [11, 12, 13, 14].

Postnikov held a permanent position at the Steklov Institute and a professorship at Moscow State University. In 1967, he organized an algebraic topology seminar at the University. Originally, it was a seminar for second-year students (like the author was at that time) where participants learned basic algebraic topology. Later, when the original participants became $\mathrm{PhD}$ students, the seminar turned into a research one. In the early 1970's, a geometric topologist A. V. Chernavskil and his students joined the seminar, and together we studied the Hauptvermutung following Casson-Sullivan-KirbySiebenmann. This benefited both teams: the geometric people learned (co)bordism theory, the homotopy people learned geometric tricks. Since mid-1970's, Chernavskiu has been a co-chair of the seminar.

The seminar has played an important role in development of algebraic topology in Moscow: many Moscow-based topologists grew up there, and most essential topological results from all over the world were reported on at seminar talks. As new participants joined the seminar, it has renewed and developed while keeping traditions and providing succession. 
In the beginning of Postnikov's graduate studies, Pontryagin suggested to Postnikov a research problem. After a while, Postnikov made some progress on the problem and decided to share it with Pontryagin. During the conversation, Postnikov realized that Pontryagin already knew everything that Postnikov was explaining to him and that, moreover, he already knew the solution. This made Postnikov very angry ("Когда я это понял, я страшно разозлился"), and he decided to stop working on that problem and work on something entirely different. (I must add though that this episode did not ruin the good relationship between Pontryagin and Postnikov.)

On the contrary, the problems that Postnikov's own PhD students got from him were definitely interesting, while the supervisor did not have any ideas of how to solve them. On the other hand, his great intuition allowed him to choose solvable problems. Furthermore, he was always open for discussions, spending a lot of time with his students. Usually he understood what you were trying to tell him very fast, and even his small comments and remarks were extremely helpful. In total, 23 Postnikov PhD students defended their PhD theses successfully. It is also worth mentioning that almost all Moscow-based algebraic topologists are either Postnikov students, or students of his students, or students of students of his students.

The first generation of Postnikov's students were students in the mid-fifties. Among them were B. Averbuch, L. Ivanovskil, S. Novikov. All the future generations of students came from and went through the seminar. Among them there are P. Akhmetiev, A. Bolibrukh, N. Gozman, A. Kharshiladze, A. Khokhlov, V. Leksin, Yu. Muranov, A. Ognikyan, A. Pajitnov, V. Pidstrigach, Yu. Rudyak, N. Saveliev, A. Szucs.

Postnikov played an enormous role in establishing the Russian topology library. Most of the books on algebraic topology that were published in Russia from the 1950's and well into the 1990's, appeared with his active participation (authorship, translation, editorship, recommendation for translation). As a good example, let me mention the collection [31] that contained translations of pivotal papers by A. Borel, R. Bott, H. Cartan, J.-P. Serre, R. Thom. This was a very important collection because in those times many of the Soviet universities (and hence scientists) were not able to access foreign literature directly. Also, Postnikov itself wrote several books at the undergraduate and graduate level, both on geometry and topology and on algebra and number theory. These are listed in the bibliography at the end of this article.

Mikhail Mikhailovich Postnikov passed away on May 27, 2004. He was active until the last days of his life. Students were delighted with his unforgettable lectures, colleagues admired his wide mathematical knowledge. He liked to travel, to play chess and bridge. He enjoyed many other things in life. Many people will thoroughly miss him.

\section{References}

[1] P. M. Akhmet'ev, D. V. Anosov, A. A. Bolibrukh, V. M. Bukhshtaber, A. V. Chernavskiŭ, V. A. Kolosov A. A. Mal'tsev, S. P. Novikov, and V. A. Smirnov, Mikhail Mikhailovich Postnikov (on his 70 birthday), Russian Math. Surveys 53 (1998), no. 2, 431-433. 
[2] V. G. Boltyanskii, R. V. Gamkrelidze, A. A. Mal'tsev, E. F. Mishchenko, S. P. Novikov and Yu. B. Rudyak, Mikhail Mikhailovich Postnikov (on his 60 birthday), Russian Math. Surveys 44 (1989), no. 6, 201-203.

[3] L. Brouwer, Über Abbildung von Mannigfaltigkeiten, Math. Ann. 71 (1912), 598.

[4] P. Hilton, G. Mislin and J. Roitberg, Localization of Nilpotent Groups and Spaces, NorthHolland Publishing Company, Amsterdam, 1975.

[5] H. Hopf, Abbildung geschlossener Mannigfaltigkeiten auf Kugeln in $n$ Dimensionen, Jahresbericht D. M. V. 34 (1926), 130-133.

[6] H. Hopf, Über die Abbildungen der dreidimensionalen Sphäre auf die Kugelfläche, Math. Ann. 104 (1931), 637-665.

[7] H. Hopf, Die Klassen der Abbildungen der $n$-dimensionalen Polyeder auf die $n$ dimensionale Sphäre, Comment. Math. Helv. 5 (1933), 39-54.

[8] S. Eilenberg, Cohomology and continuous mappings. Ann. of Math. (2) 41 (1940), 231-251.

[9] S. Eilenberg and S. MacLane, Determination of the second homology and cohomology groups of a space by means of homotopy invariants, Proc. Nat. Acad. Sci. U.S.A. 32 (1946), 277-280.

[10] S. Eilenberg and S. MacLane, Relations between homology and homotopy groups of spaces. II, Ann. of Math. (2) 51 (1950), 514-533.

[11] M. A. Evgrafov and M. M. Postnikov, A certain property of Siegel's modular group, Mat. Sb. (N.S.) 78 (120) (1969), 485-500 (in Russian).

[12] M. A. Evgrafov and M. M. Postnikov, A certain property of the Teichmüller metric, Dokl. Akad. Nauk SSSR 187 (1969), 1229-1231 (in Russian).

[13] M. A. Evgrafov and M. M. Postnikov, The asymptotics of Green's functions of parabolic and elliptic equations with constant coefficients, Mat. Sb. (N.S.) 82 (124) (1970), 3-29 (in Russian).

[14] M. A. Evgrafov and M. M. Postnikov, More on the asymptotic behavior of the Green's functions of parabolic equations with constant coefficients, Mat. Sb. (N.S.) 92 (134) (1973), 171-194, 343 (in Russian).

[15] Y. Félix, S. Halperin and J.-C. Thomas, Rational Homotopy Theory, Graduate Texts in Mathematics 205, Springer-Verlag, New York, 2001.

[16] J. Moore, Semi-simplicial complexes and Postnikov systems, in: Symposium internacional de topología algebraica, Universidad Nacional Autónoma de México and UNESCO, Mexico City, 1958, 232-247.

[17] L. S. Pontryagin, A classification of mappings of a three-dimensional complex into the two-dimensional sphere, Mat. Sb. (9) 51 (1941), 331-363.

[18] M. M. Postnikov, The classification of continuous mappings of a three-dimensional polyhedron into a simply connected polyhedron of arbitrary dimension, Doklady Akad. Nauk SSSR (N.S.) 64 (1949), 461-462 (in Russian).

[19] M. M. Postnikov, Classification of continuous mappings of an $(n+1)$-dimensional complex into a connected topological space which is aspherical in dimensions less than $n$, Doklady Akad. Nauk SSSR (N.S.) 71 (1950), 1027-1028 (in Russian).

[20] M. M. Postnikov, Determination of the homology groups of a space by means of the homotopy invariants, Doklady Akad. Nauk SSSR (N.S.) 76 (1951), 359-362 (in Russian).

[21] M. M. Postnikov, On the homotopy type of polyhedra, Doklady Akad. Nauk SSSR (N.S.) 76 (1951), 789-791 (in Russian).

[22] M. M. Postnikov, On the classification of continuous mappings, Doklady Akad. Nauk SSSR (N.S.) 79 (1951), 573-576 (in Russian). 
[23] M. M. Postnikov, Investigations in the homotopy theory of continuous mappings. I. The algebraic theory of systems. II. The natural system and homotopy type, Amer. Math. Soc. Transl. (2) 7 (1957), 1-134 (translation of Trudy Mat. Inst. Steklov. 46, Izdat. Akad. Nauk SSSR, Moscow, 1955).

[24] M. M. Postnikov, Investigations in the homotopy theory of continuous mappings. III. General theorems of extension and classification, American Mathematical Society Translations (2) 11, American Mathematical Society, Providence, R.I. 1959, 115-153 (translation of Mat. Sb. (N.S.) 40 (82) (1956), 415-452).

[25] M. M. Postnikov, Pages of a mathematical autobiography (1942-1953), in: Golden Years of Moscow Mathematics, 2nd ed., S. Zdravkovska and P. L. Duren (eds.), History of Mathematics 6, American Mathematical Society, Providence, RI; London Mathematical Society, London, 2007 (translation of Istor.-Mat. Issled. (2) No. 2 (37) (1997), 78-104, $328)$.

[26] V. A. Rohlin, A three-dimensional manifold is the boundary of a four-dimensional one, Doklady Akad. Nauk SSSR (N.S.) 81 (1951), 355-357 (in Russian).

[27] N. Steenrod, Products of cocycles and extensions of mappings, Ann. of Math. (2) 48 (1947), 290-320.

[28] J. H. C. Whitehead, On simply connected, 4-dimensional polyhedra, Comment. Math. Helv. 22 (1949), 48-92.

[29] C. Wilkerson, Classification of spaces of the same $n$-type for all $n$, Proc. Amer. Math. Soc. 60 (1976), 279-285.

[30] H. Whitney, The maps of an n-complex into an n-sphere, Duke Math. J. 3 (1937), 51-55.

[31] Fiber Spaces and Their Applications, Collection of translations, edited by V. G. Boltyanskiǔ, E. B. Dynkin, M. M. Postnikov, Izdat. Inostr. Lit., Moscow, 1958, 460 pp. (in Russian). 


\section{The list of works of M. M. Postnikov}

\section{Articles}

[1] The structure of the ring of intersections of three-dimensional manifolds, Doklady Akad. Nauk. SSSR (N.S.) 61 (1948), 795-797 (in Russian).

[2] The classification of continuous mappings of a three-dimensional polyhedron into a simply connected polyhedron of arbitrary dimension, Doklady Akad. Nauk SSSR (N.S.) 64 (1949), 461-462 (in Russian).

[3] Homology invariants of continuous mappings, Doklady Akad. Nauk SSSR (N.S.) 66 (1949), 161-164 (in Russian).

[4] Classification of the continuous mappings of an arbitrary $n$-dimensional polyhedron into a connected topological space which is aspherical in dimensions greater than unity and less than n, Doklady Akad. Nauk SSSR (N.S.) 67 (1949), 427-430 (in Russian).

[5] Classification of continuous mappings of an $(n+1)$-dimensional complex into a connected topological space which is aspherical in dimensions less than n, Doklady Akad. Nauk SSSR (N.S.) 71 (1950), 1027-1028 (in Russian).

[6] Determination of the homology groups of a space by means of the homotopy invariants, Doklady Akad. Nauk SSSR (N.S.) 76 (1951), 359-362 (in Russian).

[7] On the homotopy type of polyhedra, Doklady Akad. Nauk SSSR (N.S.) 76 (1951), 789-791 (in Russian).

[8] On the classification of continuous mappings, Doklady Akad. Nauk SSSR (N.S.) 79 (1951), 573-576 (in Russian).

[9] Definite families of functions and algebras without divisors of zero over the field of real numbers, Uspehi Mat. Nauk (N.S.) 9 (1954), 67-104 (in Russian).

[10] Investigations in the homotopy theory of continuous mappings. I. The algebraic theory of systems. II. The natural system and homotopy type, Trudy Mat. Inst. Steklov. 46 (1955), 158 pp. Translated in Amer. Math. Soc. Transl. (2) 7 (1957), 1-134 (in Russian).

[11] Homology theory of smooth manifolds and its generalizations, Uspehi Mat. Nauk (N.S.) 11 (1956), 115-166.

[12] Investigations in homotopy theory of continuous mappings. III. General theorems of extension and classification, Mat. Sb. (N.S.) 40 (1956), 415-452 (in Russian). Translated in Amer. Math. Soc. Transl. (2) 11 (1959), 115-153.

[13] Squares of classes of contrahomologies, Akad. Nauk Gruzin. SSR Trudy Tbiliss. Mat. Inst. Razmadze 22 (1956), 127-164 (in Russian).

[14] Classification of continuous mappings of an $n$-dimensional polyhedron into a connected topological space, aspherical in dimensions greater than unity and smaller than $n$, Akad. Nauk Gruzin. SSR Trudy Tbiliss. Mat. Inst. Razmadze 22 (1956), 165-202 (in Russian).

[15] Homology theory of smooth manifolds and its generalizations, Advancement in Math. 3 (1957), 503-546 (in Chinese).

[16] Cubical resolvents, Dokl. Akad. Nauk SSSR (N.S.) 118 (1958), 1085-1087 (in Russian).

[17] Limit complexes of cubic resolvents, Dokl. Akad. Nauk SSSR (N.S.) 119 (1958), 207-210 (in Russian).

[18] (with V. G. Boltjanskii) On the fundamental concepts of algebraic topology. Axiomatic definition of cohomology groups, Dokl. Akad. Nauk SSSR 133, 745-747 (in Russian). Translated as Soviet Math. Dokl. 1 (1960), 900-902. 
[19] The equivalence of cohomology theories, Trudy Mat. Inst. Steklov. 64 (1961), 165-172 (in Russian).

[20] Homotopy theory, Itogi Nauki (Algebra. Topology. 1962), 107-133. Akad. Nauk SSSR Inst. Nauchn. Informacii, Moscow (in Russian).

[21] Contemporary Riemannian geometry and its application, in: First Math. Summer School, Naukova Dumka, Kiev, 1964, 257-320.

[22] Calculation of homology (cohomology) groups and homotopy groups, Second Math. Summer School, Naukova Dumka, Kiev, 1965, 125-146.

[23] On paracompactness of cell-like polyhedra, Uspehi Mat. Nauk 20 (1965), 226-230 (in Russian).

[24] Spectral sequences, Uspehi Mat. Nauk 21 (1966), 141-148 (in Russian).

[25] On a theorem of Cartan, Uspehi Mat. Nauk 21 (1966), 35-46 (in Russian).

[26] Banach manifolds, in: Fourth Math. Summer School (Kaciveli, 1966), Naukova Dumka, Kiev, 1966 (in Russian).

[27] (with M. A. Evgrafov) A certain property of Siegel's modular group, Mat. Sb. (N.S.) 78 (1969), 485-500 (in Russian).

[28] (with M. A. Evgrafov) A certain property of the Teichmüller metric, Dokl. Akad. Nauk SSSR 187 (1969), 1229-1231 (in Russian).

[29] (with M. A. Evgrafov) The asymptotics of Green's functions of parabolic and elliptic equations with constant coefficients, Mat. Sb. (N.S.) 82 (1970), 3-29 (in Russian).

[30] (with M. A. Evgrafov) More on the asymptotic behavior of the Green's functions of parabolic equations with constant coefficients, Mat. Sb. (N.S.) 92 (1973), 171-194 (in Russian).

[31] Axiomatic homology theory and the Alexander duality theorem, in: Topics in Topology (Keszthely, 1972), Colloq. Math. Soc. János Bolyai 8, North-Holland, Amsterdam, 1974, 529-531.

[32] Localization of topological spaces, Uspehi Mat. Nauk 32 (1977), 117-181 (in Russian).

[33] (with D. V. Anosov, R. V. Gamkrelidze, E. F. Mishchenko) The mathematical work of L. S. Pontryagin, in: Modern Problems of Mathematics. Differential Equations, Mathematical Analysis and their Applications. Trudy Mat. Inst. Steklov 166 (1984), 3-17.

[34] Three-dimensional spherical forms, in: Discrete Geometry and Topology, Trudy Mat. Inst. Steklov. 196 (1991), 114-146 (in Russian). Translated in Proc. Steklov Inst. Math. 1992, 129-161.

[35] Pages of a mathematical autobiography (1942-1953), in: Golden Years of Moscow Mathematics, Hist. Math. 6, Amer. Math. Soc., 1993, 155-178.

[36] The lower Steenrod algebra, Uspekhi Mat. Nauk 49 (1994), 187-188 (in Russian). Translated in Russian Math. Surveys 49 (1994), 192-193.

[37] Naturalness and totality of cohomology operations, Trudy Mat. Inst. Steklova 224 (1999), 306-310 (in Russian). Translated in Proc. Steklov Inst. Math. 1999, no. 1 (224), 281-285.

[38] The problem of squarable lunes, translated from the Russian by Abe Shenitzer. Amer. Math. Monthly 107 (2000), 645-651.

[39] On the asymptotics of Green functions of parabolic equations, Trudy Mat. Inst. Steklova 236 (2002), 272-284 (in Russian). Translated in Proc. Steklov Inst. Math. 236 (2002), 260-272. 


\section{Books}

[1] Основы теории Галуа [Foundations of Galois theory], Gosudarstv. Izdat. Fiz.-Mat. Lit., Moscow, 1960, 124 pp.

[2] Англо-русский словарь математических терминов [English-Russian dictionary of mathematical terms], Editorial board: P. S. Aleksandrov (Chairman), L. N. Bolshev, V. S. Vladimirov, L. D. Kudrjavcev (Vice Chairman), A. F. Leontiev, S. M. Nikolskii, M. M. Postnikov, E. D. Solomencev, I. R. Shafarevich, Izdat. Inostr. Lit., Moscow, 1962, $371 \mathrm{pp}$.

[3] Магические квадраты [Magic squares], Nauka, Moscow, 1964, 84 pp.

[4] Вариачионная теория геодезических [The variational theory of geodesics], Nauka, Moscow, 1965, 248 pp.

[5] Введение в теорию Морса [Introduction to Morse theory], Nauka, Moscow, 1971, 567 pp.

[6] Аналитическая геометрия [Analytic geometry], Nauka, Moscow, 1973, 752 pp.

[7] Теорема Ферма. Введение в теорию алгебраических чисел [Fermat's theorem. Introduction to the theory of algebraic numbers], Nauka, Moscow, 1978, 128 pp.

[8] Устойчивые многочлены [Stable polynomials], Nauka, Moscow, 1981, 176 pp.

[9] Группь и алгебры Ли [Lie groups and algebras], Лекuии по геометрии, Семестр V [Lectures in geometry, Semester V], Nauka, Moscow, 1982, 448 pp.

[10] Введение в теорию алгебраических чисел [Introduction to algebraic number theory], Nauka, Moscow, 1982, 240 pp.

[11] Лекции по алгебраической топологии [Lectures in algebraic topology], Основы теории гомотопий [Elements of homotopy theory], Nauka, Moscow, 1984, 416 pp.

[12] Лекиии по алгебрачческой топологии [Lectures in algebraic topology], Теория гомотопий клеточных пространств [Homotopy theory of cell complexes], Nauka, Moscow, 1985, 336 pp.

[13] Аналитическая геометрия [Analytic geometry], Лекиии по геометрии. Семестр I [Lectures in geometry. Semester I], 2nd ed., Nauka, Moscow, 1986, 416 pp.

[14] Линейная алгебра [Linear algebra], Лекиии по геометрии. Семестр II [Lectures in geometry. Semester II], 2nd ed., Nauka, Moscow, 1986, 401 pp.

[15] Гладкие многообразия [Smooth manifolds], Лекции по геометрии. Семестр III [Lectures in geometry. Semester III], Nauka, Moscow, 1987, 479 pp.

[16] Дифференциальная геометрия [Differential geometry], Лекиии по геометрии. Семестр IV [Lectures in geometry. Semester IV], Nauka, Moscow, 1988, 496 pp.

[17] Риманова геометрия [Riemannian geometry], Лекиии по геометрии. Семестр V [Lectures in geometry. Semester V], Faktorial, Moscow, 1998, 496 pp.

[18] Geometry VI. Riemannian geometry, translated from the 1998 Russian edition by S. A. Vakhrameev, Encyclopaedia of Mathematical Sciences 91, Springer-Verlag, Berlin, 2001, xviii+503 pp.

\section{Book translations}

[1] Расслоеннье пространства и их приложения [Fiber spaces and their applications], collection of translations, edited by V. G. Boltyanskii, E. B. Dynkin, M. M. Postnikov, Izdat. Inostr. Lit., Moscow, 1958, 460 pp. 
[2] И. Капланский, Введение в дифференциальную алгебру [I. Kaplansky, An introduction to differential algebra], translated from the English by G. I. Kleinerman, edited by M. M. Postnikov, Izdat. Inostr. Lit., Moscow, 1959, 85 pp.

[3] А. Картан, С. Эйленберг, Гомологическая алгебра [H. Cartan, S. Eilenberg, Homological algebra], translated from the English by E. G. Shulgeifer, edited by M. M. Postnikov, Izdat. Inostr. Lit., Moscow, 1960, 510 pp.

[4] М. Балдасари, Алгебраические многообразия [M. Baldassarri, Algebraic varieties], translated from the English by Yu. I. Manin, edited by M. M. Postnikov, Izdat. Inostr. Lit., Moscow, 1961, 315 pp.

[5] Р. Мошер, М. Тангора, Когомологические операции и их приложения в теории гомотопий [R. Mosher, M. Tangora, Cohomology operations and applications in homotopy theory], translated from the English by M. M. Postnikov, Mir, Moscow, 1970, 287 pp.

[6] П. Габриель, М. Цисман, Категории частных и теория гомотопий [P. Gabriel, M. Zisman, Categories of fractions and homotopy theory], translated from the English by M. M. Postnikov, Mir, Moscow, 1971, 295 pp.

[7] Дж. Уайтхед, Новейшие достижения в теории гомотопий [G. Whitehead, Recent advances in homotopy theory], translated from the English by A. Yu. Geronimus, edited by M. M. Postnikov, Mir, Moscow, 1974, 128 pp.

[8] К. Рурк, Б. Сандерсон, Введение в кусочно линейную топологию [C. Rourke, B. Sanderson, Introduction to piecewise-linear topology], translated from the English by I. V. Saveliev, edited by M. M. Postnikov, Mir, Moscow, 1974, 208 pp.

[9] Дж. Бордман, Р. Фогт, Гомотопически инвариантные алгебраические структурь на топологических пространствах [J. Boardman, R. Vogt, Homotopy invariant algebraic structures on topological spaces], translated from the English by S. N. Malygin, edited by M. M. Postnikov, Mir, Moscow, 1977, 408 pp.

[10] И. Мадсен, Р. Милграм, Классифицирующие пространства для перестроек и кобордизмов многообразий [I. Madsen, R. Milgram, The classifying spaces for surgery and cobordism of manifolds], translated from the English and with an appendix by Yu. B. Rudyak, translation edited by M. M. Postnikov, Mir, Moscow, 1984, 279 pp. 
